\title{
DYNAMIC BATTERY MODEL AND STATE OF CHARGE ESTIMATION
}

\author{
S. Wijewardana, R. Vepa, M. H. Shaheed *
}

School of Engineering and Material Science, Queen Mary, University of London, London, E14NS, UK.

\begin{abstract}
Mathematical modeling and the dynamic simulation of battery storage systems can be challenging and demanding due to the nonlinear nature of the battery chemistry. This paper introduces a new dynamic battery model, with application to state of charge estimation, considering all possible aspects of environmental conditions and variables. The aim of this paper is to present a suitable convenient, generic dynamic representation of rechargeable battery dynamics that can be used to model any Lithium-ion rechargeable battery. The proposed representation is used to develop a dynamic model considering the thermal balance of heat generation mechanism of the battery cell and the ambient temperature effect including other variables such as storage effects, cyclic charging, battery internal resistance, state of charge etc. The results of the simulations have been used to study the characteristics of a Lithium-ion battery and the proposed battery model is shown to produce responses within $98 \%$ of known experimental measurements.
\end{abstract}

Keywords: Unscented Kalman Filter, Dynamic modeling, Temperature effect, Hybrid vehicles, Simulation, Lithium-ion.

* Corresponding author: Tel: +442078823319; E-mail address: m.h.shaheed@ qmul.ac.uk. 


\section{I.INTRODUCTION}

Efficiency, novelty and the latest technology in hybrid cars pose the greatest attraction among commuters in the automotive world. More than just the fuel economy, hybrid vehicles offer many green advantages. A small increase in fuel economy makes a large difference in emissions over many years. Battery designs play an important role in these vehicles, and the price per kilowatt-hour varies according to battery type as mentioned in Smith [1].

The Lithium-ion (Li-ion) batteries have attracted the popularity among many battery types to be used in hybrid electric vehicles. Dynamic modeling that accounts for all aspects of the battery life cycle such as self-discharging, gassing effect, diffusion processes, acid stratification, state of charge, over voltage stabilization etc. is quite an involved process as outlined by Zoroofi [2].

Broadly, battery models may be classified as circuit based models and electrochemical models. However the distinction is only superficial, as all electro-chemical models can be represented by equivalent circuits, if one permits the circuit elements to be active or regenerative elements rather than just passive circuit elements. Furthermore the circuit elements may be non-linear functions of the internal state of the battery. The basic circuit model is the Thevenin equivalent circuit of the battery and its extensions as discussed in Vepa [3]. In these approaches one establishes a linear model of the relationship between the terminal voltage and the current available to the application. The polarization losses are expressed as nonlinear voltage losses. An alternate approach is to assume a linear relationship between the rate of change of the terminal voltage and the rate of change of the state of charge (SOC), which is usually the key metric of interest. The proportionality constant could be assumed to be locally linear or nonlinear. The SOC is defined as the percentage of the completely 
extractable charge capacity remaining in the battery. The SOC indicates the amount of electrical energy remaining in a battery. A key performance parameter is an accurate estimate of the SOC as and when it is being used and is important both for the battery application designers as well as for the battery users. An accurate indication of the battery SOC during the runtime allows the user to ensure that the battery is neither over-charged or under-discharged, resulting not only in the optimum use of the power available but also in a longer battery life. The methods of estimating the batter SOC can be broadly classified as:

i) Discharge test under controlled conditions to determine the capacity remaining at any instant after a specific loss of capacity;

ii) Ampere hour counting or charge counting (initial SOC must be known and involves estimation of the current loss);

iii) Based on the relation between the open circuit voltage and SOC;

iv) Model based estimation;

v) Heuristic interpretation of measured data (electrolyte properties and other).

Several of the above methods rely on test data and the usual tests carried out on a battery are:

i) Open Circuit Voltage Test to determine the relationship between the terminal voltage, the terminal current and the state of charge;

ii) Hybrid Pulse Test: Hybrid Pulse Power Characterisation (HPPC) profiles with constant current discharge and charge pulses with rest periods or pauses with no charging or discharging are used to measure battery performance characteristics;

iii) Federal Urban Driving Schedules Tests (FUDS): FUDS is a typical dynamic driving cycle and is usually used in the USA to verify the usefulness and the accuracy of the battery models. 
Most measured data is characterised by uncertainties and there are usually five classes of uncertainties to consider: i) measurement uncertainty, ii) algorithmic uncertainty, iii) environmental uncertainty, iv) model parameter uncertainty and v) model dynamics uncertainty or un-modelled dynamics. Thus the first step is usually to extract the model parameters by applying suitable model identification and parameter estimation methods to the measured data as discussed by Birkl and Howey [4]. One can adopt either a time domain approach, such as the method of Alavi, Birkl and Howey [5] which can then be used directly to simulate the battery dynamics (see for example Thanagasundram et al [6]) or a circuit based approach as in Howey et al [7]. One could also adopt a direct approach and identify the SOC characteristic from the measured data as was done by Li, Chattopadhyay and Ray [8].

A novel approach to battery modeling can be found in Sepasi, Ghorbani and Liaw [9] and in Xia et al [10]. Here, we investigate to present a cell level battery model which incorporates the most significant parameters that have been mentioned previously. As mentioned earlier, circuit based electrical models are very useful and complex enough to represent the electrochemical behaviour within the cells that describe the dynamics of the electron transfer and energy dissipation. Zhang et al [11], Thanangasundram et al [6] and MathWorks [12] used the open circuit voltage to estimate the state of charge of the battery. Erdinc, Vural, Uzunoglu [13] employed the battery internal resistance as a function of SOC when modeling discharging/charging characteristics. Storage and cyclic effects also were added to the internal resistance. The dynamics of the SOC, is given by Erdinc, Vural, Uzunoglu [13] and is,

$$
\frac{d \operatorname{SOC}(T)}{d n}=k_{1} n+k_{2} .
$$

The temperature effect on the battery system was modeled along with the rate of change of SOC. In equation (1), $n$ is the cycle number, $k_{1}$ and $k_{2}$ are constants. The 
variable $T$ identifies the operating temperature of the battery. The equation (1) was based on a semi empirical formulation for variation of SOC at the negative electrode with the cycling effect confined to two specific temperatures of $25^{\circ} \mathrm{C}$ and $50^{\circ} \mathrm{C}$.

When modeling the effect of battery temperature, experiments by Tan, Mao and Tseng [14] demonstrate that it is essential to consider both the change in the ambient temperature $T_{a m b}$ due to environmental conditions and the rise in internal battery cell temperature $T_{\text {Cell }}$ due to chemical reactions in the electrolyte and the electron transfer effect. Although most experiments are conducted at a constant temperature (see for example Chen and Rincon-Mora [15]), for electric vehicles (EV) especially, the rise in the ambient temperature due to a hot external environment as well as the heat emission from an automobile engine can affect the battery performance severely as mentioned by Kroeze and Krein [16]. To account for all the performance characteristics, the effects of both the ambient and internal battery cell temperature have to be explicitly considered. Though, the change is not substantial, temperatures above a critical temperature can affect the battery performance. Simulation results show that this critical temperature for a lithium-ion battery $T_{\text {Cell }}$ is about $42{ }^{\mathrm{O}} \mathrm{C}$.

In this paper a new dynamic Li-ion battery model is proposed and validated. One of the key features of this paper is that, it presents a dynamic battery model that is accurate and consistent not only at a constant cell temperature but also at varying ambient and internal cell temperatures. The other contributions made in this paper are categorized as follows a) a new table of coefficients for the variable model parameters of $R_{\mathrm{int} S}, R_{T S}, R_{T L}, C_{T S}, C_{T L}$ (which will be described in section- IV). b) The implementation of thermal energy balance model that can be described as a reduced order thermal model. c) The introduction of two new variable voltage sources $\Delta E(T)$ 
and $\Delta V_{C h e}(T)$ that depend on the temperature. The change of $\Delta E(T)$ represents the equilibrium potential correction of the battery open-circuit voltage, that depends on the temperature and the amount of active material available in the electrodes, that can be specified in terms of state of discharge (SOD) (Panday and Bansal [17]). $\Delta V_{C h e}(T)$ represents the voltage increase due to temperature effect on the electrolyte chemistry (film formation, non-uniform ions distribution, non-uniform heat generation rate, conductivity difference in the electrolyte due to hot regions, diffusion and overvoltage stabilization). d) Due to temperature and SOC change during simulation time (see for example $[12,18]$ ) the variable discharge/charge current has been included in the model to get the accurate battery terminal voltage. e) A new formulation representing the SOC variation due to varying temperature.

The paper is organized as follows. Section II briefly describes the existing dynamic battery models. The new dynamic battery model is described in section III. The thermal energy balance equation, with our contributions to the new dynamic battery model is given in section IV. The final non-linear state equations of the model are summarised in section $\mathrm{V}$. The comparison of the model with the experimental results at $25^{\circ} \mathrm{C}$ is presented in section VI. The effect of the variable temperature on the model is discussed in section VII. The application to SOC estimation is reviewed and discussed in section VIII. The conclusion is given in section IX.

\section{EXISTING DYNAMIC BATTERY MODELS}

Li-ion batteries have been considered for modeling as they have been getting greater attention from the researchers than $\mathrm{NiMH}$ and Lead-acid batteries due to their higher specific energy, specific density, durability and lower self-discharge rate (See for example Panday and Bansal [17]). In addition, Li-ion batteries have earned a good 
reputation for their excellent life cycle with no memory effects. These physical characteristics make the Li-ion batteries preferable for next generation of hybrid electric vehicles [13]. The parameters required for these models have been defined in the nomenclature.

Various classes of mathematical models of battery performance based on the chemistry of the electrolyte, the SOC, the electro-chemical behaviour and the thermal effects have been presented by Zoroofi [2], MathWorks Inc. [12], Tremblay and Dessaint [18], Gallo et al [19], Huria [20], Tan, Mao and Tseng [14], Chen and Rincon-Mora [15], Bhide and Shim [21]. All of these models could be used, in part, although none of the above models have ventured to include all of the effects that are of importance for representing the battery dynamics for electric vehicle and hybrid energy system applications. In this paper we have integrated the battery chemistry, the evolution of the SOC, the electro-chemical dynamics and the thermal effects into one single model.

\section{NEW DYNAMIC BATTERY MODEL}

A dynamic battery model that can accommodate both electrical and thermal properties which is suitable to apply for any hybrid energy systems is sought. The equivalent electrical circuit for such a model is shown in fig.1(a) which has the following five parts from left to right:

a) Source voltage $V_{O C}$, in parallel with the $R_{\text {self }}(T, n, S O C)$ which is identified as $R_{\text {self }}$ representing the battery self-discharge feature due to storage effects which is a function of temperature $T, S O C$ and the cycle number $n$. The cycle number $n$ signifies the number of cycles the battery is charged or discharged. 
b) Battery internal resistance which is modelled by two resistances in series $R_{\text {int } S}$ and $R_{C Y C}$ representing the electrolyte resistance and the resistance change due to the number of charging and discharging cycles the battery has undergone.

c) Two $R C$ circuit loops with $R C$ elements given by, $R_{T S}, C_{T S}$ and $R_{T L}, C_{T L}$ which represents the short and long transient effects of the battery. The transient effects arise due to double-layer formation at the electrode/solution interface (Thanagasundram et al [6]). The capacitances $C_{T S}$ and $C_{T L}$ represent the electrical polarization capacitance and the diffusion capacitances. Transient response of the battery is influenced by double layer diffusion capacitance when the rates of reactions are high.

d) The parameters $R_{\mathrm{int} S}, R_{\mathrm{TS}}, R_{T L}, C_{T S}, C_{T L}$ and SOC/SOD usually depend on temperature. Their dependence is included separately in the model by using mathematical relationships that will be discussed later in this section.

e) Finally, the other temperature dependent characteristics are included into the model by the addition of two variable voltage sources $\Delta E(T)$ and $\Delta V_{C h e}(T)$.

A selection of batteries with different electrolytes which are associated with different chemistries and temperature effects and are dissimilar, have been considered for the electro-chemical modelling. Due to the gassing effect and film formation at the electrodes the electron transfer rates will be changed. Temperature change can influence these effects and alter the equilibrium potential or open circuit voltage (Thanagasundram et al [16]). These effects have been included in this study.

\section{THERMAL MODEL}

The thermal model presented here is based on the general energy balance equations used in Bernardi, Pawlikowski and Newman [22]. Heat is produced in batteries from three fundamental sources: activation (interfacial kinetics), concentration (species 
transport), and Ohmic (Joule heating from the movement of charged particles) losses. The proposed thermal model assumes the natural convection conditions and the heat generation and heat dissipation throughout the electrolyte as uniform, although models of uneven heat generation of the type discussed by $\mathrm{Wu}$ et al [23] could be incorporated into the analysis. The model parameters and the chemistry between anode, cathode and the separator have not considered separately but taken as average values. The generation rates of reversible and irreversible heat during charge and discharge are taken as equal with the same SOC and current rate. The temperature distribution is taken as symmetrical with respect to centre line in the battery cell. Initial battery cell temperature is assumed to be equal to the ambient temperature. Detailed description in this context can also be found in $\mathrm{Wu}, \mathrm{Li}$ and Zhang [24]. The equation for the total heat generation rate given by equation (7) in [24] is used to model the thermal model. Hence the energy balance equation according to [22] can be written as given below (2):

$$
\begin{aligned}
& \Delta T=\left(T_{\text {Cell }}-T_{\text {amb }}\right) \\
& \frac{d T_{\text {Cell }}}{d t}=\frac{1}{m C_{p}}\left[i\left\{V_{\text {bat }}-V_{O C}\right\}+\frac{T_{\text {Cell }}}{F} \Delta S \Phi-A \alpha \Delta T\right]-A \lambda \Delta T-A \sigma \varepsilon\left(T_{\text {Cell }}^{4}-T_{\text {amb }}^{4}\right)
\end{aligned}
$$

The parameter $\alpha$ is normally identified as the heat transfer coefficient for forced cooling or in literature sometimes it is defined as the convective heat exchange coefficient. The symbol $\Phi$ is the current density $\left(\mathrm{A} / \mathrm{cm}^{2}\right)$. The parameter $F$ is defined as the Faraday constant. The parameter $\sigma$ is the Stefan- Boltzmann constant. The effect of other two parameters, $\lambda$ which is identified as the conductive heat coefficient and $\varepsilon$ which is defined as the radiation coefficient are neglected due to minimal effects. The combined entropy change $\Delta S$, can also be taken as zero as in Viswanathan et al [25]. 
Hence the equation (3) can be simplified to equation (4) as given below ([2, 21]):

$$
\frac{d T_{\text {Cell }}}{d t}=\frac{1}{m C_{p}}\left[i\left\{V_{b a t}-V_{O C}\right\}-A \alpha \Delta T\right]
$$

where $m$ is the unit mass. The temperature effect on activation, diffusion polarization and due to different electrolyte chemistries is modelled by the parameter $\Delta V_{C h e}(T)$ which is a function of the temperature.

The parameter $\Delta V_{C h e}(T)$ is modeled by the polynomial as given by the equation (5):

$$
\Delta V_{\text {Che }}=\beta w \exp ^{(1 / t)} \Delta T+\left.\frac{d V_{\text {che }}}{d T}\right|_{T=T_{\text {Cell }}}\left(C_{\text {Che }}+C_{\text {Che1 }} \Delta T\right)(1+\beta) \Delta T
$$

where $T_{\text {Cell }}$ is the battery internal temperature. Though it is not uniform here we assumed the $T_{\text {Cell }}$ to be uniform within the cell and $T_{a m b}$ is the operating temperature of the battery. The parameters $\beta, w, C_{C h e}$ and $C_{C h e 1}$ are all constants that depend on the properties of the electrolyte, the anode and the cathode materials. The first derivative $d V_{\text {che }} / d T$ is defined as the effective voltage gradient. $\Delta E(T)$ can be modelled by the equation as given in (6), as,

$$
\Delta E(T)=\left.\left(1+C_{E 1} \Delta T\right) \frac{d V r}{d T}\right|_{T=T_{C e l l}} \Delta T
$$

The first derivative $d V_{r} / d T$ is defined as the voltage gradient and is assumed to be constant for the temperature range. Table-I gives the parameters and constants derived from simulation results [indicated in the table as 'sim'] and directly from the literature or estimated from the literature [indicated in the table as 'Lit'].

By considering the two $R C$ parallel loops the following mathematical relationships can be deduced by using Kirchhoff's law ((7), (8) and (9)).

$$
\frac{d V_{j}}{d t}=\frac{i}{C_{T j}}-\frac{V_{j}}{R_{T j} C_{T j}}, j=1,2 .
$$


In (7),

$C_{T j}=C_{T L}, R_{T j}=R_{T L}$, for $j=1$,

and:

$C_{T j}=C_{T S}, R_{T j}=R_{T S}$, for $j=2$.

$V_{\text {OC } 0}=V_{\text {bat }}+i\left(R_{\text {int } s}+R_{c y c}\right)+V_{1}+V_{2}$

The parameters $V_{1}$ and $V_{2}$ represents the voltages across the two capacitors $C_{\mathrm{TS}}$ and $C_{\mathrm{TL}}$. The open circuit voltage $V_{O C}$ is then expressed as,

$$
V_{\text {OC }}=V_{O C 0}+\Delta E(T)+\Delta V_{C h e}(T) .
$$

The battery terminal current is related to the battery voltage by the relation,

$i=C_{b a t} \dot{V}_{b a t}$.

If the battery voltage is assumed to be related to the state of charge by the relation,

$V_{b a t}=k * S O C+d$,

we obtain the model equation in differential form,

$\dot{V}_{b a t}=k * S \dot{S O C}$

In the non-linear case we begin with a locally linear model of the form given by equation (11b) which can then be integrated to give a nonlinear model of the battery voltage and SOC relationship. Using equations (11a) and (11b) in equations (8) and (10) respectively gives,

$$
\begin{aligned}
& V_{O C 0}=k * S O C+d+i\left(R_{\mathrm{ints}}+R_{c y c}\right)+V_{1}+V_{2} \\
& S \dot{O C}=i / k C_{b a t}
\end{aligned}
$$


Where $V_{O C 0}=V_{O C 0}(S O C, n, T)$ is the open circuit voltage under standard conditions or at the room temperature and the state of charge is given by the charge counting equation,

$$
S O C=S O C_{i n i}-\int\left(i / C_{\text {usable }}\right) d t
$$

Using equation (13) we can write:

$$
S O C=S O C_{i n i} /\left(1+k C_{b a t} / C_{\text {usable }}\right)
$$

Hence the constant $k$ is defined by,

$$
k=C_{\text {usable }}\left(S O C_{i n i}-S O C\right) / C_{b a t} S O C
$$

The complete set of equations defining the battery dynamics above, do not include a noise model. We will include two noise sources: The first with the current in the form of a process noise and modify the current equation to,

$$
\dot{i}=w_{1} \text {. }
$$

The second noise source is in the measured open circuit voltage, $V_{O C}$,

$$
V_{O C}=V_{O C 0}+\Delta E(T)+\Delta V_{C h e}(T)+v_{n}
$$

The usable battery capacity $C_{\text {usable }}$ changes depending on the storage time and temperature. When the temperature increases the usable capacity decreases. An excellent detailed description on $C_{\text {usable }}$ with the effect of temperature can be found in Spotnitz [26] and in Plangklang and Pornharuthai [27]. From Valerie, Johnson and Pesaran [28] it was noted that from $0^{\circ} \mathrm{C}$ to $60^{\circ} \mathrm{C}$ the usable capacity decreases roughly about $4 \%$ for a simulation time of one hour. Hence, the change of this parameter is included in the model by using a capacity correction factor (CCF) which is reasonable, instead of representing the variation with a polynomial equation that fits into manufacturers' data. The CCF is generally calculated by using the operating temperature and the time of storage which is normally measured in months as it gives 
an indication of the per cent loss in the capacity fading. The capacity fading can also be calculated using the equations given in Nasar and Unnewehr [29]. From the Lithium-ion Rechargeable Batteries technical data handbook [30], discharge capacity remains constant from $22^{\circ} \mathrm{C}$ to $45^{\circ} \mathrm{C}$. The discharge capacity decreases linearly from $22^{\circ} \mathrm{C}$ to $0^{\circ} \mathrm{C}$. This can be taken as a negative gradient of $2 \mathrm{mAh}$ per degree Celsius. For Li-ion though the discharging temperature range is permissible between $-20^{0} \mathrm{C}$ to $60^{\circ} \mathrm{C}$, the charging temperature range is only recommended from $0^{\circ} \mathrm{C}$ to $45^{\circ} \mathrm{C}$ and not below freezing temperature as also given in BU-Charging [31].

The battery equivalent internal impedance $\left(R_{\text {int } S}+R_{c y c}\right)$ contributes to the instantaneous drop in the battery terminal voltage as given by Zoroofi [2]. As discussed earlier, the passive circuit elements $R_{T S}$ and the $C_{T S}$ represent the fast dynamics associated with the reaction kinetics between the electrical charges at the electrode's surface barrier and the charges in the electrolyte. The $R_{T S}$ is the charge transfer resistance and the capacitance $C_{T S}$ models the electrochemical double layer capacitance. The $R_{T L}$ and the $C_{T L}$ circuit elements represent the slower dynamics of the cell in the order of hours. They are more representative of the battery chemistry related to the diffusion processes in the electrolyte and active electrode material. All of these parameters are functions of the ambient temperature, electrolyte temperature $T_{C e l l}, S O C$, and the battery current. The components of $R C$ networks $R_{T S}, C_{T S}, R_{T L}$ and $C_{T L}$ are responsible for the short and the long-term transients in the battery internal impedance. Ideally this equivalent circuit should include two additional parallel loops to account for hysteresis phenomena of the battery. When the additional loops are included, both the short hysteresis and the long hysteresis behaviour of the battery can be predicted. These temperature effects were included in the mathematical model 
presented by the equations (4) and (5) defined earlier. The parasitic behaviour of the capacitors which is considered to be minimal and hence it is being ignored in this model but could be included if and where necessary. Models of the hysteresis of the type discussed by Huria, Ludovici and Lutzemberger [32] and Windarko and Choi [33] could be easily included if and when necessary.

The self- discharging effect which is modeled by a loop containing $V_{O C}$ source parallel with $R_{\text {self }}(T, n, S O C)$ as given by fig.1(a). Panday and Bansal [17] have presented a method and a formula for simulating the self-discharge current through the $R_{\text {self }}(T, n, S O C)$ in terms of the self-discharging current $i_{S_{-} D}$ through $R_{\text {self }}(T, n, S O C)$. There the parameter $C_{p}$ is the initial battery capacity before storage. The symbol $T$ signifies the variable temperature, $R_{\text {gas }}$ is the gas constant and $K_{O}$ is a reaction rate constant. The reaction rate is given by the Arrhenius equation in terms of $T, R_{\text {gas }}, K_{O}$ and the activation energy, $E_{A}$ and was used by Panday and Bansal [17].

However, as the resistance of $R_{\text {self }}(T, n, S O C)$ which is identified as $R_{\text {self }}$, is very high compared to $\left(R_{\text {int } S}+R_{C Y C}\right)$, and therefore the current passes through $R_{\text {self }}$ can be taken as zero. Hence, we can neglect the voltage drop across the $R_{\text {self }}$ resistor.

The effect of $R_{\text {int } S}, B, C_{T S}$, and $C_{T L}$ due to the battery SOC can be calculated as given in [13] and expressed by the generic equation, (19):

$$
R_{c g}(S O C)=C_{g 1} \exp \left(-C_{g 2} S O C\right)+C_{g 3} \exp \left(C_{g 4} S O C\right)
$$

The capacitances are limited to small positive values when the SOC is zero. The constants $C_{g 1}, C_{g 2}, C_{g 3}$ and $C_{g_{4}}$ are presented in the Table II. The effect of the temperature on $R_{\mathrm{int} S}(S O C, T)$ can be given by the polynomial and is defined as,

$$
R_{\mathrm{int} S}(S O C, T)=-1.399 \times 10^{-5} T^{2}-0.0002768 \times T+R_{\mathrm{int} S-r e f}(S O C) .
$$


In (20) the parameter $R_{\text {int } S-r e f}(S O C)$ is the battery internal resistance at $0^{0} \mathrm{C}$, $R_{\text {int } S-r e f}(S O C)=0.146$ for the Panasonic 17500 Li-Ion-battery with a nominal voltage of $3.7 \mathrm{~V}$ for a discharge current of $830 \mathrm{mAh}$, at $25^{\circ} \mathrm{C}$ and $S O C=1$ )

The SOC change due to temperature has been proposed by many researchers using Arrhenius equation, [17, 29]. According to experimental data available from Valerie, Johnson and Pesaran [28] and from He et al [34] it was noted that the SOC decreases with the increase of temperature and the simulation time. The SOC can decrease up to $50 \%$ from $100 \%$, with the increase of temperature from $15^{\circ} \mathrm{C}$ to $60^{\circ} \mathrm{C}$. It was also noted from the literature that SOC change can be taken as linear within the above temperature range. Experimental/simulation data shows that the temperature increase due to one hour discharge at (1C rate) is less than $0.2^{0} \mathrm{C}$ (Spotnitz [26]) and hence the variation due to simulation time can sometimes be neglected in modeling if the simulation time is one hour or less. Using experimental data from Zhu et al [35] and considering the $S O C$ to be a maximum when the temperature is nearly ambient, a polynomial equation is proposed in this paper to calculate initial SOC due to temperature effect and is given by the polynomial equation (21):

$$
\operatorname{SOC}_{\text {ini }}=1-0.000442(\Delta T+0.714932)^{2}, \Delta T=T_{\text {Cell }}-T_{a m b} .
$$

From equation (3), assuming the inputs are slowly varying, it follows that the battery cell temperature $T_{C e l l}$ is related to the time by the equation:

$$
T_{\text {cell }}=\frac{\left[i\{\text { Vbat }-V o c\}+A \alpha T_{a m b}\right]}{A \alpha}\left(1-\exp \left(-\frac{A \alpha}{m C_{p}} t\right)\right) .
$$

Equation (13) was verified by fitting the simulation data with two exponential functions and it was found that one of them was indeed very slowly varying and 
almost constant. The battery open circuit voltage, which is dependent on battery SOC can be calculated by using equation (23) as given in [13].

$$
\begin{aligned}
& V_{O C 0}=-1.031 \times \exp (-35 \times S O C)+3.685+0.2156 \times S O C-0.1178 \times S_{S O C}^{2} \\
& +0.321 \times S_{O C C}^{3} .
\end{aligned}
$$

Battery internal resistance change due to cyclic effect is assumed to be given by the empirical equation, (24), as in [13]:

$$
R_{c y c}=\zeta \times n^{\lambda}
$$

In equation (24), $n$ is the discharge/charge cycle number and $\xi$ is a constant $(\xi=0.0015)$. The constant $\lambda$ is normally taken to be equal to $1 / 2$ according to [13]. The SIMULINK simulation diagram of the system shown in fig. 1(a), is shown in fig. 1(b) and includes all of the equations represented by (1)-(16) and (19)-(24).

\section{FINAL NONLINEAR STATE SPACE 5-STATE MODEL EQUATIONS}

The final nonlinear, 5-state, state space equations defining the dynamic battery model are summarized below.

i) Battery Current:

$$
i=w_{1} \text {; }
$$

ii) State of Charge (SOC):

$$
S \dot{C} C=i / k C_{b a t}
$$

iii) Fast \& Slow Polarization Over-potential:

$$
\frac{d V_{j}}{d t}=\frac{i}{C_{T j}}-\frac{V_{j}}{R_{T j} C_{T j}}, j=1,2 .
$$

iv) Cell Temperature: 


$$
\frac{d T_{\text {Cell }}}{d t}=\frac{1}{m C_{p}}\left[i\left\{V_{b a t}-V_{O C}\right\}-A \alpha \Delta T\right]
$$

v) Open Circuit Output Voltage:

$$
V_{O C}=V_{O C 0}+\Delta E(T)+\Delta V_{C h e}(T)+v_{n} ;
$$

vi) Open Circuit Output Voltage without film \& chemistry:

$$
V_{\text {OC } 0}=i\left(R_{\mathrm{ints}}+R_{c y c}\right)+k * S O C+V_{1}+V_{2}+d ;
$$

vii) Voltage due to electrode film formation:

$$
\Delta E(T)=\left.\left(1+C_{E 1} \Delta T\right) \frac{d V r}{d T}\right|_{T=T \text { Cell }} \Delta T ;
$$

viii) Voltage due to electrolyte electron transfer chemistry:

$$
\Delta V_{\text {Che }}=\beta \operatorname{Kexp}^{(-1 / t)} \Delta T+\left.\frac{d V_{\text {che }}}{d T}\right|_{T=T \text { Cell }}\left(C_{\text {Che }}+C_{\text {Che } 1} \Delta T\right)(1+\beta) \Delta T .
$$

\section{THERMAL MODEL COMPARISON WITH EXPERIMENTAL RESULTS}

\section{AT $25{ }^{\circ} \mathrm{C}$}

The experimental results published by Tan, Mao and Tseng [14] and Thanagasundaram et al [6] have been used in this paper to test and validate the model. The Li-ion TCL PL-383562, which has the capacity of $850 \mathrm{mAh}$ is used to validate the model and the parametric constants generated by using the Panasonic $17500 \mathrm{Li}$ ion-battery is also used (as the nominal voltages are same).

The current pulse tests at discharging currents of 80, 160, 320 and $640 \mathrm{~mA}$ had been used. The nominal capacity (the average capacity) of the battery is $800 \mathrm{mAh} \pm$ $5 \%$ at a discharge rate of $0.2 \mathrm{~A}$ in $0.2 \mathrm{C}$ (5 hour discharge) and it was discharging at a temperature of $25^{\circ} \mathrm{C}$ with more than 300 cycles. The battery can be charged at a charge rate of $0.8 \mathrm{~A}$ in $1 \mathrm{C}$ (1 hour charge). When discharging at $1.6 \mathrm{~A}$ it will last 2 
hours (0.5C). The manufacturers specify the charge cut off voltage as $4.2 \mathrm{~V}$ and the discharge cut off voltage as $2.75 \mathrm{~V}$. The results from the simulation of the proposed model are compared with the experimental results published by Chen and RinconMora [15]. Fig. 2, 3 and 4 illustrate open-circuit voltage and the error variations of the battery parameters with the $S O C$ at $750 \mathrm{~mA}$ battery current. Percentage error was calculated for each graph by using the formula given by equation (25) which is embedded into MATLAB code and finally calculating the mean percentage error, which can be simply calculated by using MATLAB command.

$$
\operatorname{Err}(\%)=100 \frac{\left(1-\left(a b s\left(V_{O C_{-} s i m}-V_{O C_{-} E x p}\right)\right.\right.}{V_{\text {OC_sim }}} .
$$

The parameters $V_{O C_{-} \text {sim }}$, and $V_{O C_{-} \text {Exp }}$ represents the data arrays of simulation results and the experimental results respectively. These comparisons confirm the validity of the new model and the responses within $98 \%$ of known experimental measurements.

From fig.3, the simulation and experimental data the percentage accuracy was 98.215\%. Selected battery data is stated for reference: CC/CV charge at 4.2V, 1C $+25^{0} \mathrm{C}$ and CC discharge at .2C to $2.75 \mathrm{~V}$; Nom. Volt.: 3.75, Nom. Capacity: $190 \mathrm{mAh}$ to $1800 \mathrm{mAh}, \operatorname{Max}^{\mathrm{m}}$ current: $1 \mathrm{C}$; $\operatorname{Max}^{\mathrm{m}}$ voltage: $4.2 \mathrm{~V}$.

The new model was tested for known experimental data, published by Thanagasundaram et al [6] and the the response calculated by using Matlab (and equation(25)) happened be within 98.7\% accuracy (fig.4). The battery data used in [6] is as follows: Lithium Manganese Oxide Battery; nominal voltage 3.6CC: 750mA, nominal capacity 2200mAh. 


\section{THE VARIABLE TEMPERATURE EFFECT: MODEL COMPARISON WITH EXPERIMENTAL RESULTS}

Simulation and battery data results so far presented assumed that the temperature was constant during the time of simulation and the operating temperature was $25^{\circ} \mathrm{C}$ during the time of battery charging/discharging. The parameter values $R_{\mathrm{int} S}, R_{T S}, R_{T L}$, $C_{T S}, C_{T L}$ and SOC changes according to charging or discharging. They are assumed to be invariant with respect to charging or discharging Plangklong [27].

The Panasonic Lithium-ion batteries, CGR18650 and CGR17500 were chosen for this part of the simulations with temperature effect and the manufacturer's specifications are recorded for reference. The charge and discharge conditions of the batteries are also provided by Tan, Mao and Tseng [14], Chen and Rincon-Mora [15], and the handbooks [36] and [37]. For CGR18650: Charge Conditions: constant voltage/constant current, $4.2 \mathrm{~V}, 910 \mathrm{~mA}$ (max), 2 hours, at $20^{\circ} \mathrm{C}$ as specified by the manufacturers. Discharge conditions: Constant current (CC) 260 mA. For CGR17500: Charge conditions: CV/CC, 4.2V, $550 \mathrm{~mA}$ (max), 2 hours, $20^{\circ} \mathrm{C}$. Discharge Conditions: Constant current $780 \mathrm{~mA}$. The specifications and experimental data from Tan et al [14] for Li-ion battery CGR17500 have been used in this model to validate the thermal and electrical model.

At high temperatures chemical reactions require less activation energy as the atoms can move faster. This will result in a higher cell voltage. However, optimum rate of reaction occurs between $38^{\circ} \mathrm{C}$ to $42^{0} \mathrm{C}$. Increase in temperature beyond the optimum limit along with the ambient temperature can be detrimental to the battery life. Conversely, at low temperatures, intercalation and de-intercalation requires higher activation energy for the chemical reactions. In Li-ion batteries, this occurrence can cause less lithium ion participation in the cell mechanism resulting in temporary loss 
of capacity. Fig. 5 shows the manufacturers' data and the new model simulation results with varying temperatures. Fig. 5(b) shows the battery discharge capacity versus the cell voltage with diffierent constant ambient temperatures during the time of simulation and also with varying temperaturetures from $-20^{\circ} \mathrm{C}$ to $60^{\circ} \mathrm{C}$ during the time of simulation which confirms the model as correct.

As it can be seen from fig.6, the simulation curves coincide with the experimental data plots and hence, the model can be validated as an accurate model which has both electrical and thermal properties. In addition, the experimental data reported in a NREL report which was presented by Valerie et al [28] where it was confirmed that at $40^{\circ} \mathrm{C}$, the $O C V$ is nearly the same as its value at the ambient temperature in addition to the information given in BU-Charging/discharging [31]. The reported data also conforms to the new simulations and the data plots as given by fig.6.

\section{APPLICATION TO SOC ESTIMATION}

It may be observed first the equations (17), (13), (7), (4) and (18) may be expressed as two decoupled sets of equations. Introducing the variable, $\Delta V_{O C}=V_{O C}-V_{b a t}$, and using equation (12), equation (18) is expressed as,

$$
\Delta V_{O C}=i\left(R_{\text {int } s}+R_{c y c}\right)+V_{1}+V_{2}+\Delta E+\Delta V_{C h e} .
$$

Equation (13) is now completely decoupled from equations (17), (7), (4) and (18).

To demonstrate a typical, novel application of the model, we describe briefly a typical application of the battery model to SOC estimation. Our aim is to use a simple extended Kalman filter and yet obtain results similar to those obtained with adaptive filtering or by using the unscented Kalman filter as has been done by He et al [34], Plett [38] and Tian et al [39]. Early reviews of SOC estimation based on measured 
impedance data was presented by Rodrigues, Munichandraiah and Shukla [40] and by Piller, Perrin and Jossen [41]. A comparative study of various state estimation algorithms is presented by Barillas et al [42], Li et al [43] and Zou et al [44]. While an enhanced Coulomb counting method has been proposed by Ng et al [45], the current focus is on model based estimation as demonstrated by He et al [46] and Sun et al [47]. Xing et al [48] have included temperature effects in their model although it does not include temperature dynamics. Both real time (He, Xiong and Guo [49]) and adaptive estimation (Waag and Sauer [50]) have been pursued. To deal with the specific issues that arise with SOC estimation, a multi-model approach is proposed by Wang, Zhang and Chen[51], a dual time scale approach by Dai et al [52] as well as multi-scale approaches by Hu, Youn and Chung [53] and by Xiong et al [54] to Kalman filtering, have been proposed. Because, the model equations naturally decouple into two sets, a dual Kalman filtering approach has been proposed by Walder et al [55] based on the use of two decoupled Kalman filters which has since been improved by Campestrini et al [56] and Lee et al [57]. There have also been some novel applications of estimation to original battery configurations presented by Zhong et al [58], Pérez et al [59] and Fares, Meyers and Webber [60].

The estimation of SOC in this paper also exploits the decoupled nature of the model equations. It is based on the extended Kalman filter and is done by writing the model equations, (17), (7), (4) and (28) in the form:

$$
\begin{aligned}
& \dot{i}=w_{1} ; \\
& \dot{V}_{T S}=\frac{1}{C_{T S}}\left(i-\frac{V_{T S}}{R_{T S}}\right), \dot{V}_{T L}=\frac{1}{C_{T L}}\left(i-\frac{V_{T L}}{R_{T L}}\right), \\
& \frac{d T_{C e l l}}{d t}=\frac{-1}{m C_{p}}\left[i \Delta V_{O C}+A \alpha \Delta T\right],
\end{aligned}
$$




$$
\begin{aligned}
& \dot{R}_{T S}=w_{2}, \\
& \dot{R}_{T L}=w_{3}, \\
& \dot{C}_{T S}=w_{4}, \\
& \dot{C}_{T L}=w_{5},
\end{aligned}
$$

where $w_{n}, n=1,2 \ldots 5$ are Gaussian White noise disturbances of known intensity and $\Delta V_{O C}$ is given by equation (28). The measurement is given by, $\Delta V_{O C m}=\Delta V_{O C}+v_{n}$, where $v_{n}$ is a Gaussian White noise disturbance of known intensity. Equations (29) and the measurement are used to estimate the current $i$ and $\Delta V_{O C}$ initially. The estimated SOC is then obtained by integrating equation (13), which is expressed as,

$$
S \hat{O} C=\hat{i} / k C_{b a t} .
$$

The equations are linearized so the methodology of the extended Kalman filter is applied at every step of the simulation. The extended Kalman filter has the predictorcorrector structure and corrects the estimate based on the innovation or new information in the measurement. It must be added that the estimation could be extended to the case when $k$ and $d$ are nonlinear functions of the state and need to be updated in real time.

The estimate of the $S O C$ at the room temperature of $30^{\circ} \mathrm{C}$ is compared in the first of subplot of fig. 7 to the simulation over a time frame of 600 seconds and the error is less than $2 \%$. Also shown in the second subplot of fig. 7 , is a comparison of the estimated and measured $V_{O C}$. The estimate of the current $\hat{i}$ based on an initial constant current input (discharging) of $i=-5$ amps is shown in fig. 8. All of the battery parameters necessary for the simulations were first calculated from the 
manufactures data sheets. The estimate error is less than $0.02 \%$. In this simulation the temperature was assumed to be a constant. Similar curves may also be obtained when the temperature is dynamically measured and filtered.

\section{CONCLUSIONS}

In DC power source applications such as hybrid electric vehicles (HEVs), monitoring algorithms use current and voltage measurements to estimate the battery's SOC and available power. The literature says that the SOC change has no effect on the generation rate of the ohmic heat contributed by the contact resistance between electrode and current collector The open circuit voltage and electrode surface concentration nonlinearities, film formation at electrodes, side reactions and the temperature effect cause dynamic modeling difficulties for real time applications. The new battery model presented here could provide satisfactory results and offer good solutions for many questions that simulation engineers face with. The decoupled approach to SOC estimation presented in the preceding section is a novel and unique feature of this paper and such a decoupled approach has not been used previously. It is currently being used in developing a real time SOC estimation methods based on both nonlinear unscented and extended Kalman filtering in order to explicitly demonstrate that the extended Kalman filter gives comparable results to the unscented Kalman filter in a real-time scenario. Thus the models developed in this paper are proving to be extremely useful both for simulation and estimation studies.

\section{REFERENCES}

[1] Smith AK. Electrochemical modeling, estimation and control of lithium-ion batteries. PhD Thesis in Mechanical Engineering, The Graduate School, The Pennsylvania State University, December, 2006; 1-15 
[2] Zoroofi S. Modeling \& simulation of vehicular power systems. MSc Thesis, submitted in partial fulfilment of requirements for the degree Master of Science, Department of Energy and Environment, Division of Power Engineering Chalmers University of Technology, Goteborg, Sweden, 2008; Chapter-5:23-36

[3] Vepa R. Dynamic Modeling, Simulation and Control of Energy Generation, Lecture notes in Energy, Springer, ISBN 978-1-4471-5400-6, 2013.

[4] Birkl CR and Howey DA. Model identification and parameter estimation for LiFePO4 batteries, Proc. of Conf. on Hybrid and Electric Vehicles 2013 (HEVC 2013), London. 2013; 2.1-2.1.

[5] Alavi SMM, Birkl CR and Howey DA. Time-domain fitting of battery electrochemical impedance models, Journal of Power Sources; 2015; 288:345-352.

[6] Thanangasundram S, Arunachala R, Makinejad K, Teutsch T. A cell level model for battery simulation. European Electric Vehicle Congress; Brussels, Belgium, 2012; 20-22.

[7] Howey DA, Yufit V, Mitcheson PD, Offer GJ, and Brandon NP. Impedance measurement for advanced battery management systems, Pro. of the 2013 World Electric Vehicle Symposium and Exhibition (EVS27), Barcelona. 2013; 1-7.

[8] Li Y, Chattopadhyay P, Ray A. Dynamic data-driven identification of battery state-of-charge via symbolic analysis of input-output pairs, Appl. Energy. 2015; 155:778-790.

[9] Sepasi S, Ghorbani R, Liaw BY. A novel on-board state-of-charge estimation method for aged Li-ion batteries based on model adaptive extended Kalman filter, Journal of Power Sources; 2014; 245:337-344. 
[10] Xia B, Chen C, Tian Y, Sun W, Xu Z, Zheng W. A novel method for state of charge estimation of lithium-ion batteries using a nonlinear observer. Journal of Power Sources; 2014; 270:359-366.

[11] Zhang T. The Economic Benefits of Battery Energy Storage System in Electric Distribution System. M.Sc Thesis in Electrical and Computer Engineering, Submitted at Worcester Polytechnic Institute, 2013:12-28

[12] The MathWorks Inc. Generic Battery Model. [Online], Available, http:// www.mathworks.co.uk, Accessed on, 16 Dec 2013.

[13] Erdinc O, Vural B, Uzunoglu M. A dynamic Lithium-ion battery model considering the effects of temperature and capacity Fading. IEEE International Conference on Clean Electrical Power. 2009; Capri, 383-386.

[14] Tan YK, Mao JC, Tseng KG. Modeling of Battery Temperature effect on Electrical Characteristic of Li-ion Battery in Hybrid Electric Vehicles. Power Electronics and Drive Systems Conference; Singapore. IEEE 2011;637-642.

[15] Chen M, Rincon-Mora GA. Accurate Electrical Battery Model Capable of Predicting Runtime and I-V Performance. IEEE Transactions on Energy Conversion.2006; 21(2):504-511.

[16] Kroeze RC, Krein PT. Electrical Battery Model for Use in Dynamic Electric Vehicle Simulations. IEEE Power Electronics Specialists Conference; 2008; PESC, University of Illinois at Urbana-Champaign Dept. of Electrical and Computer Engineering, Urbana, IL 61801. 2008:1336-1342.

[17] Panday A, Bansal HO. Temperature dependent circuit-based modeling of high power Li-Ion battery for plug-in hybrid electrical vehicles, ICATE 2013; IEEE Paper Identification Number-85:1-7 
[18] Tremblay O, Dessaint LA. Experimental Validation of a Battery Dynamic Model for EV Applications. EVS24 International Battery, Hybrid and Fuel Cell Electric Vehicle Symposium, World Electric Vehicle Journal.2009;3:1-9.

[19] Gallo D, Landi C, Luiso M, Morello R. Optimization of Experimental Model Parameter Identification for Energy Storage Systems. Energies. 2013; 6(9):45724590

[20] Huria T. Rechargeable lithium battery energy storage systems for vehicular applications -Land Vehicles and Transport Systems. PhD Dissertation Universita Di PISA Engineering School. 2012;34-43

[21] Bhide S, Shim T. Novel Predictive Electric Li-Ion Battery Model Incorporating Thermal and Rate Factor Effects. IEEE TRANSACTIONS ON VEHICULAR TECHNOLOGY. 2011;60(3):819-829.

[22] Bernardi D, Pawlikowski E, Newman J. A General Energy Balance for Battery Systems. Journal of the Electrochemical Society. 1985: 132(1):5-12.

[23] Wu B, Yufit V, Marinescu M, Howey DA, Martínez-Botas RF and Brandon NP., Modelling and measurement of uneven heat generation in lithium-ion battery packs. 10th Symposium on Fuel Cell and Battery Modelling and Experimental Validation (ModVal 10) Stuttgart, 18-20 March 2013.

[24] Wu B, Li Z, Zhang J. Thermal design for the pouch-type large-format Lithiumion batteries, I. Thermo-Electrical modeling and origins of temperature nonuniformity. Journal of the Electrochemical Society. 2015;162(1):181-191

[25] Viswanathan VV, Choi D, Wang D, Xu W, Towne S. Effect of entropy change of lithium intercalation in cathodes and anodes on Li-ion battery thermal management. Journal of Power Sources. 2010; 195:3720-3729. 
[26] Spotnitz R. Simulation of capacity fade in Lithium-ion batteries. Journal of Power Sources. 2003;113:81-100.

[27] Plangklang B, Pornharuthai P. Mathematical Model and Experiment of Temperature Effect on Discharge of Lead-Acid Battery for Systems in Tropical Area. Energy and Power Engineering. Scientific Research. 2013;5:43-49.

[28] Valerie H, Johnson AA, Pesaran A. Temperature-Dependent Battery Models for High-Power Lithium-Ion Batteries. National Renewable Energy Laboratory (NREL), USA, 17th Electric Vehicle Symposium Montreal, Canada. 2000;6-17.

[29] Nasar A, Unnewehr LE. Electromechanics and Electric Machines, Second Ed., John Wiley \& Sons Inc. USA, 1993;

[30] Lithium-ion Batteries (Panasonic) Technical Handbook’ 99. [Online] available at: http://www.master-instruments.com.au/files/data_sheets/Lithium/Panasonic\%20Lithium\%20Ion\%20Hanbook.pdf. accessed: 22 June 2015:14-25

[31] BU-Charging at high Low Temperatures. [Online] available at http://batteryuniversity.com/learn/article/charging_at_high_and_low_temperatures. Accessed: 22 Jan 2015:1-7

[32] Huria T, Ludovici G, Lutzemberger G. State of charge estimation of high power lithium iron phosphate cells, Journal of Power Sources. 2014; 249:92-102.

[33] Windarko A and Choi J. SOC Estimation Based on OCV for NiMH Batteries Using an Improved Takacs Model. Journal of Power Electronics. 2010; 10(2):181186.

[34] He Z, Gao M, Wang C, Wang L, Liu Y. Adaptive State of Charge Estimation for Li-Ion Batteries Based on an Unscented Kalman Filter with an Enhanced Battery Model. Energies 2013;(6):4134-4151. 
[35] Zhu C, Li X, Song L, Xiang L. Development of a theoretically based thermal model for Lithium-ion battery pack. Journal of Power Sources. 2013; 223:155-164.

[36] Sony Corp. Lithium Ion Rechargeable Batteries Technical Handbook. [Online]. Available: www.sony.com.cn/products/ed/battery/download.pdf. Accessed: 19 Oct 2014;17-29.

[37] Temperature and discharge rate. [Online] Available: http://www.mpoweruk.com/soc.htm. Accessed: 23 Jan 2015;1-5

[38] Plett G. Sigma-point Kalman filtering for battery management systems of LiPBbased HEV battery packs: Part 1: Introduction and state estimation. J. Power Sources. 2006;161:1356-1368.

[39] Tian Y, Xia B, Sun W, Xu Z, Zheng W. A modified model based state of charge estimation of power Lithium-ion batteries using unscented Kalman filter. Journal of Power Sources. 2014;270:619-626.

[40] Rodrigues S, Munichandraiah N, Shukla AK. A review of state-of-charge indication of batteries by means of a.c. impedance measurements. Journal of Power Sources. 2000; 87:12-20.

[41] Piller S, Perrin M, Jossen A. Methods of state-of-charge determination and their application. Journal of Power sources. 2001; 96:113-120.

[42] Barillas JK, Li J, Günther C, Danzer MA. A comparative study and validation of state estimation algorithms for Li-ion batteries in battery management systems. Appl. Energy. 2015; 155: 455-462

[43] Li J, Barillas JK, Guenther C, Danzer MA. A comparative study of state of charge estimation algorithms for $\mathrm{LiFePO}_{4}$ batteries used in electric vehicles. Journal of Power Sources. 2013; 230:244-250. 
[44] Zou Z, Xu J, Mi C, Cao B and Chen Z. Evaluation of Model Based State of Charge Estimation Methods for Lithium-Ion Batteries. Energies. 2014; 7:50655082.

[45] Ng KS, Moo C-S, Chen Y-P, Hsieh Y-C. Enhanced coulomb counting method for estimating state-of-charge and state-of-health of lithium-ion batteries. Appl. Energy. 2009; 86:1506-1511.

[46] He Y, Liu X, Zhang C, Chen Z. A new model for State-of-Charge (SOC) estimation for high-power Li-ion batteries. Appl. Energy. 2013; 101:808-814.

[47] Sun F, Xiong R, He H, Li W, Aussems JEE. Model-based dynamic multiparameter method for peak power estimation of lithium-ion batteries. Appl. Energy. 2012; 96:378-386.

[48] Xing Y, He W, Pecht M, Tsui KL. State of charge estimation of lithium-ion batteries using the open-circuit voltage at various ambient temperatures. Appl. Energy. 2014;113:106-115.

[49] He H, Xiong R, Guo H, Online estimation of model parameters and state-ofcharge of LiFePO4 batteries in electric vehicles. Appl. Energy. 2012; 89:413-420.

[50] Waag W, Sauer DU. Adaptive estimation of the electromotive force of the lithium-ion battery after current interruption for an accurate state-of-charge and capacity determination. Appl. Energy. 2013; 111:416-427.

[51] Wang Y, Zhang C, Chen Z. A method for state-of-charge estimation of Li-ion batteries based on multi-model switching strategy. Appl. Energy. 2015;137:427434.

[52] Dai H, Wei X, Sun Z, Wang J. Online cell SOC estimation of Li-ion battery packs using a dual time-scale Kalman filtering for EV applications. Appl. Energy. 2012;95:227-237. 
[53] Hu C, Youn BD, Chung J. A multiscale framework with extended Kalman filter for lithium-ion battery SOC and capacity estimation. Appl. Energy. 2012; 92:694704.

[54] Xiong R, Sun F, Chen Z, He H. A data-driven multi-scale extended Kalman filtering based parameter and state estimation approach of lithium-ion polymer battery in electric vehicles. Applied Energy. 2014; 113:463-476.

[55] Walder G, Campestrini C, Lienkamp M and Jossen A. Functionality and behaviour of an dual Kalman filter implemented on a modular battery-management system. Conference on Future Automotive Technology (CoFAT 2013): Focus Electromobility, 2013.

[56] Campestrini C, Walder G, Jossen A, Lienkamp M. Temperature Influences on State and Parameter Estimation Based on a Dual Kalman Filter. Conference on Future of Automotive Technology (CoFAT 2014) 2014.

[57] Lee S, Kim J, Lee J, Cho BH. State-of-charge and capacity estimation of lithiumion battery using a new open-circuit voltage versus state-of-charge. Journal of Power Sources. 2008; 185:1367-1373.

[58] Zhong L, Zhang C, He Y, Chen Z. A method for the estimation of the battery pack state of charge based on in-pack cells uniformity analysis. Appl. Energy. 2014; 113:558-564.

[59] Pérez G, Garmendia M, Reynaud JF, Crego J, Viscarret U. Enhanced closed loop State of Charge estimator for lithium-ion batteries based on Extended Kalman Filter. Appl. Energy. 2015; 155:834-845.

[60] Fares RL, Meyers JP, Webber ME, A dynamic model-based estimate of the value of a vanadium redox flow battery for frequency regulation in Texas. Appl. Energy. 2014; 113:189-198. 